\title{
Pattern-Avoidance in Binary Fillings of Grid Shapes (short version)
}

\author{
Alexey Spiridonov \\ Department of Mathematics, Massachusetts Institute of Technology, Cambridge, MA 02139, USA
}

\begin{abstract}
A grid shape is a set of boxes chosen from a square grid; any Young diagram is an example. This paper considers a notion of pattern-avoidance for 0-1 fillings of grid shapes, which generalizes permutation pattern-avoidance. A filling avoids some patterns if none of its sub-shapes equal any of the patterns. We focus on patterns that are pairs of $2 \times 2$ fillings. For some shapes, fillings that avoid specific $2 \times 2$ pairs are in bijection with totally nonnegative Grassmann cells, or with acyclic orientations of bipartite graphs. We prove a number of results analogous to Wilfequivalence for these objects - that is, we show that for certain classes of shapes, some pattern-avoiding fillings are equinumerous with others.
\end{abstract}

Résumé. Une forme de grille est un ensemble de cases choisies dans une grille carrée; un diagramme de Young en est un exemple. Cet article considère une notion de motif exclu pour un remplissage d'une forme de grille par des 0 et des 1, qui généralise la notion correspondante pour les permutations. Un remplissage évite certains motifs si aucune de ses sous-formes n'est égale à un motif. Nous nous concentrons sur les motifs qui sont des paires de remplissages de taille $2 \times 2$. Pour certaines formes, les remplissages évitant certaines paires de taille $2 \times 2$ sont en bijection avec les cellules de Grassmann totalement positives, ou bien avec les orientations acycliques de graphes bipartis. Nous démontrons plusieurs résultats analogues à l'équivalence de Wilf pour ces objets — c'est-à-dire, nous montrons que, pour certaines classes de formes, des remplissages évitant un motif donné sont en nombre égal à d'autres remplissages.

Keywords: pattern-avoidance, filling, grid shape, Le-diagram, acyclic orientation, Young diagram

\section{Introduction}

This short version of the paper omits many proofs, examples, counterexamples, and generally has less depth than [7]. Please refer to that paper for the details.

\subsection{Pattern-avoidance of fillings}

Perhaps the best-known example of pattern avoidance is defined for permutations. Let $S_{n}$ be the set of permutations of $[n]=\{1,2, \ldots, n\}$. A permutation $\sigma \in S_{n}$ avoids $\tau \in S_{k}$ if there is no set of indices $1 \leq i_{1} \leq \cdots<i_{k} \leq n$ such that

$$
\pi\left(i_{\tau^{-1}(1)}\right)<\pi\left(i_{\tau^{-1}(2)}\right)<\cdots<\pi\left(i_{\tau^{-1}(n)}\right) .
$$

1365-8050 @ 2008 Discrete Mathematics and Theoretical Computer Science (DMTCS), Nancy, France 
In other words, if we take the $j$ th largest value of $\left\{\pi\left(i_{l}\right)\right\}$ and replace it by $j$ in $\pi\left(i_{1}\right), \pi\left(i_{2}\right), \ldots, \pi\left(i_{k}\right)$ for all $1 \leq j \leq k$, we will not get $\tau$ 's word $\tau(1), \tau(2), \ldots, \tau(k)$ for any index set $\left\{i_{l}\right\}$.

Let $S_{n}(\sigma)$ be the set of permutations of $[n]$ that avoid $\sigma$. Permutations $\sigma$ and $\tau$ are Wilf-equivalent if $\left|S_{n}(\sigma)\right|=\left|S_{n}(\tau)\right|$ for all $n$. For more details on Wilf equivalence, and further references, see [8].

The permutation matrix of $\sigma$ is a matrix $P_{\sigma}=\left(p_{i j}\right)$ with $p_{i j}=1$ if $\sigma(i)=j$, and $p_{i j}=0$ otherwise. In terms of these matrices, the permutation $\sigma \in S_{n}$ avoids $\tau \in S_{k}$ if no $k \times k$ minor of $P_{\sigma}$ equals $P_{\tau}$. If we draw the permutation matrices with lines separating rows and columns, then permutations are just special cases of $0-1$ fillings of square shapes.

Thus, pattern-avoidance generalizes naturally to fillings of shapes, as follows: a filling $F$ avoids a filling $G$ if no minor of $F$ equals $G$ (both the shapes and fillings must agree). Wilf-equivalence also translates to this context - two patterns $p_{1}$ and $p_{2}$ are equivalent if $p_{1}$-avoiding fillings are equinumerous with $p_{2}$-avoiding fillings.

\subsection{Genesis of the problem}

Permutations are special fillings of special shapes. In Subsection 1.5, we cite several other generalizations of permutations as pattern-avoiding fillings. All those papers restrict their attention to special shapes (rectangles and Young diagrams). In some, the fillings, too, have extra restrictions (fixed row/column sums).

In contrast, this paper considers arbitrary fillings of arbitrary shapes. However, our patterns are quite special - we require that a filling avoid a pair of $2 \times 2$-fillings. We made this choice because of some results by Alex Postnikov [6]. We will restate them in terms of $2 \times 2$ pattern pairs:

1. Fillings of Young diagrams $\lambda$ that avoid the pattern pair $(: \circ \mid: \bullet)($ where $\circ=0$ and $\bullet=1$ ) are exactly the $\lrcorner$-diagrams. The latter are in bijection with totally nonnegative Grassmann cells, which are defined in [6] as elements of a particular cellular decomposition of $G r_{k, n}^{t n n}$. This $G r_{k, n}^{t n n}$ denotes, in turn, elements of the Grassmannian with nonnegative Plücker coordinates.

2. Fillings of Young diagrams $\lambda$ that avoid $\left({ }_{0}^{\circ} \|_{\circ}^{\circ}\right)$ are acyclic orientations of the diagram's bipartite graph $G_{\lambda}$ (rows and columns are vertices, boxes are edges). According to [6], the following objects associated with $\lambda$ are equinumerous: acyclic orientations of $\left.G_{\lambda},\right\lrcorner$-diagrams on $\lambda$, totally nonnegative Grassmann cells inside the Schubert cell $\Omega_{\lambda}$, and several others.

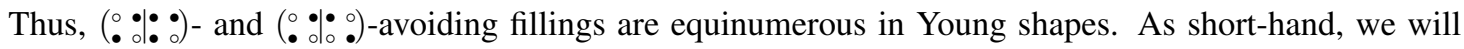

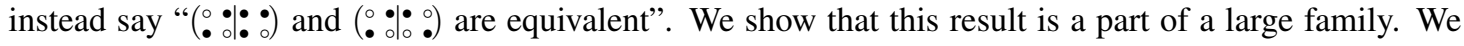
study several other pattern pairs, and show that the above equivalences hold with much weaker conditions on the shapes.

\subsection{Related work}

\lrcorner -diagrams were first used by Alex Postnikov [6] and Lauren Williams [10] to count totally nonnegative Grassmann cells. In [6], Postnikov proved the equivalences described above. Williams and Steingrímsson introduced permutation tableaux - a special kind of $\lrcorner$-diagrams - in [9], and showed that they capture many enumerative properties of permutations. Alexander Burstein ([1], pages 10-11) gave a simple bijection between $\lrcorner$-diagrams and ( $:::::)$-avoiding fillings of Young shapes. Recently, Matthieu Josuat-Vergès

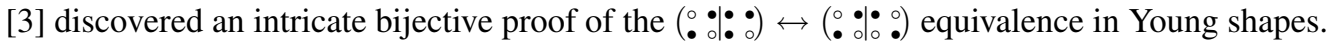




\subsection{Contribution}

This paper takes a structural approach to the problem of pattern equivalence. The main goal is to classify the equivalences of $2 \times 2$ pattern pairs, and the shapes, in which these equivalences hold.

The essential notations and definitions are in Section 2 Section 3 is a high-level discussion of the problem, our results, and what they mean. In Section 4 , we extend Postnikov's proof of the $(.: 0 \mid: 0) \leftrightarrow$ $\left(\begin{array}{c}0 \\ 0\end{array} 0^{\circ}\right.$ o) equivalence to far more general shapes, and then apply these ideas to other pattern pairs in Sections 5 and 6 Section 7 briefly describes two nice, and very different bijections, which together give tight descriptions of 6 more (not counting complements) pattern pair equivalences.

This paper, due to space constraints, has few proofs (they are in [7]). The largest number of pages here goes towards extending Williams's and Postnikov's results to shapes as general as possible. This is important, as it explains the language needed to frame further results. For the results that are completely new, only the main ideas, sufficient to reconstruct the proofs, are stated here.

This paper does not finish the classification of pattern pairs. However, it makes a substantial step forward. Firstly, it introduces the principal actors - we show that 21 out of 64 (after identifying complements) pattern pairs are involved in interesting equivalences in "nice" shapes. Computational experiments suggest that these patterns are the ones most frequently involved in equivalences. Secondly, we prove several equivalences tying the 21 patterns together. A few of these equivalences seem to be in the broadest terms possible, some have bijective proofs, but many need improvements. However, the broad outlines of the classification are now visible, and the remaining details should be a matter of time.

\subsection{Other related results}

In [8], Zvezdelina Stankova does not explicitly mention pattern-avoiding fillings. However, the main relation of that paper is shape-Wilf-ordering $\preceq_{s}$, which can be rephrased in terms of fillings. Let $\tau$ and $\sigma$ be permutations; then $\sigma \preceq_{s} \tau$ iff for every Young shape $\lambda$, the number of $0-1$ fillings with exactly one 1 in each row and column, which avoid $P_{\sigma}$, is at most the number of such fillings avoiding $P_{\tau}$. The key point here is that, just as in our definition, every cell of the square pattern $P_{\sigma}$ must be inside the Young shape to match.

In [2], Vít Jelínek studies a problem related to Stankova's. He works with $0-1$ fillings of rectangular shapes. However, instead of constraining all row and column sums to be 1 (that would make a permutation, of course), he allows an arbitrary fixed sum for each row and column. In our terms, his main result is about equivalent patterns (recall — this means "fillings avoiding them are equinumerous"). He shows that permutations of a fixed order $\leq 3$ are all equivalent, when restricted to fillings with a fixed multiset of row and column sums.

Several enumerative results about pattern-avoiding fillings are due to Kitaev, Mansour, and Vella [5], and Kitaev [4]. In both papers, the shapes are rectangles, and the fillings are binary. They consider all nontrivial patterns up to size $2 \times 2$. The first paper counts, for each of these 56 described patterns, the number of fillings of an $m \times n$ rectangle, which avoid it. It defines two notions we also use: pattern complement (see Fact 2.12), and pattern symmetry (Subsection 3.1). The second paper finds equivalences between patterns consisting of multiple fillings of 3-cell shapes. The author studies rectangles that avoid 2,3 , or 4 patterns simultaneously, and gives equivalence classes of tuples of 3-cell fillings in each case. The main approach, like in [4], is to explicitly count the pattern-avoiding fillings. 


\section{Basic Definitions}

\subsection{Shapes and fillings}

Definition 2.1. An $m \times n$-grid shape $S$ is a subset of boxes selected from a $m \times n$ 2-dimensional square grid. From now on, we will call these simply shapes.

Definition 2.2. The graph $G_{S}$ of an $m \times n$-shape $S$ is a bipartite graph on $m+n$ vertices. The first part of $G_{S}$, with $m$ vertices, corresponds to the rows of $S$. The second part, with $n$ vertices, corresponds to the columns. There is an edge in $G_{S}$ between row $i$ and column $j$ iff $S$ has a box in that position.

Definition 2.3. A filling $F$ of a shape $S$ places $\circ$ or $\bullet$ (alternative notations: $\circ$ or $\times, 0$ or 1 ) in every cell of the shape:

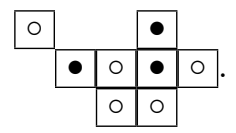

Notation 2.4. Let $c$ be a cell of a shape $S$ or filling $F$. Then, $S \backslash c$ and $F \backslash c$ denote the shape or filling with the cell removed.

Definition 2.5. Deleting some rows and columns from a shape or filling makes a minor. They are specified with a row set and column set. Here is the $\{1,2\} \times\{1,3,4\}$ minor of the filling above:

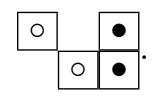

The minors of $S$ are exactly the induced subgraphs of $G_{S}$.

Definition 2.6. A $k \times k$-step in a graph consists of moving from a cell $c$ to another cell $d$ so that both are in the same $k \times k$ minor with all $k^{2}$ cells present. A shape is $k \times k$-connected if one can get from any cell to any other cell by a sequence of $k \times k$-steps. For the purpose of $2 \times 2$ pattern avoidance, it will be enough to prove theorems for $2 \times 2$-connected shapes. Such shapes have a 4 -cycle-connected $G_{S}$ - one can get from any edge to any other edge by walking along adjacent edges of 4-cycles.

Definition 2.7. A grid shape $S$ is horizontally connected if, after removing the shape's empty columns, the cells in every row form a single, unbroken block. Vertical connectivity is analogous. A shape is connected if it is both horizontally and vertically connected. This neither implies, nor is implied by $k \times k$-connectivity.

\subsection{Patterns}

Our definition of patterns for fillings generalizes standard permutation pattern-avoidance. A permutation $\sigma \in S_{n}$ can be represented as a "rook diagram". We take an $n \times n$ shape and fill it with os, except for a - in each cell $i, j$ such that $\sigma(i)=j$. The permutation 43152 contains 132 , namely 152 in positions 345 . Correspondingly, the $\{1,2,5\} \times\{3,4,5\}$ minor of the rook diagram is equal to

$$
\begin{array}{|l|l|l|}
\hline \bullet & 0 & 0 \\
\hline \circ & 0 & \bullet \\
\hline \circ & \bullet & 0 \\
\hline
\end{array}
$$

A permutation is 132 -avoiding if and only if its rook diagram avoids this minor. The generalization of such patterns to general shapes and fillings is simple: 
Definition 2.8. A pattern $p$ is a filling of a shape. A filling $F$ contains the pattern $p$ if some minor of $F$ equals $p$.

The natural question is to characterize the number of fillings of a fixed shape $S$ that avoid (or contain) certain $p$. We will focus on a specialization of this problem.

Definition 2.9. A $2 \times 2$-pattern pair, further called pattern pair, or $p p$ for short, is an unordered pair of $2 \times 2$ patterns. For example: $\left(\begin{array}{:}\circ \\ \bullet\end{array}\right.$ $p_{2}$. We will call $F$ a $P$-avoiding filling, or a $P$-paf for short.

Fix a shape $S$ and a pattern pair $P$. In this paper, we will not count the number of fillings of $S$ that avoid $P$ (although that is an interesting question, see [4, 5]). Instead, we will study the equivalence of pps:

Definition 2.10. $P_{1}=\left(p_{1}^{1} \mid p_{2}^{1}\right)$ and $P_{2}=\left(p_{1}^{2} \mid p_{2}^{2}\right)$ are equivalent, denoted $P_{1} \leftrightarrow P_{2}$, if the fillings that avoid $P_{1}$ are equinumerous with $P_{2}$-pafs.

Now it is clear why we may prove theorems only for $2 \times 2$-connected shapes:

Remark 2.11. A $2 \times 2$-disconnected shape contains a pattern iff one of its components does. A special case is when the component has exactly one cell. We call such cells detached.

The first observation about equivalent pps is that we can take a "complement" of any pp, by replacing

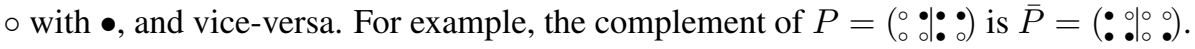

Fact 2.12. Given a shape $S$ and a pp $P, P$-pafs are equinumerous with $\bar{P}$-pafs. The bijection is obvious: $F$ is a P-paf iff $\bar{F}$ is a $\bar{P}$-paf.

Since $P$ and $\bar{P}$ are necessarily equivalent, we will identify every pp with its complement. Now, in order to enumerate all pps, we will number the single patterns. The pattern $p=\begin{array}{lll}a & b \\ c & d\end{array}$ will be assigned number $n(p)=a+2 b+4 c+8 d=d c b a_{2}$, where $\circ$ is 0 and $\bullet$ is 1 . Then, $n(\bar{p})=15-p=1111_{2}-d c b a_{2}$ is the number of its complement. For consistency, we will write pps as $\left(p_{1} \mid p_{2}\right)$ with $n\left(p_{1}\right)<n\left(p_{2}\right)$.

There are $2^{4}=16$ single patterns, and consequently $\frac{16 \cdot 15}{2}=120$ pattern pairs. There are 8 selfcomplementary pps, which have $n\left(p_{1}\right)+n\left(p_{2}\right)=15$. So, after identifying complements, we are left with $\frac{120-8}{2}+8=64$ classes of pattern pairs.

\section{Classification of Pattern Pair Equivalences}

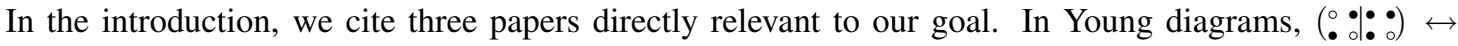

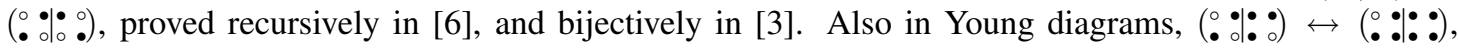
proved bijectively on pages $10-11$ of [1].

The main point of this paper is that these results are part of a much bigger picture. The work began when the author wrote a computer program to count pattern-avoiding fillings of shapes, and found a plethora of other equivalences.

\subsection{Symmetry and complements}

Some of these equivalences were simple consequences of symmetry. The equivalence-preserving symmetries on both patterns and shapes are generated by row order reversal and transposition (e.g. column reversals are row reversals conjugated by transposition). As you read the paper, keep in mind that $\left(\begin{array}{l}0 \\ \bullet\end{array} \cdot \bullet\right.$ 
is invariant under both maps. Symmetry has two distinct effects on pattern equivalence. Firstly, if one $\mathrm{pp}$ is mapped to another by a transformation $T$, then these pps will be equivalent in all shapes that have a $T$-symmetry. Most shapes are not symmetric, so these equivalences, although easy to list, usually do not apply. Secondly, if the equivalence $A \leftrightarrow B$ is known to hold in shape class $C$, then $T(A) \leftrightarrow T(B)$ holds in shape class $T(C)$. Taking complements, we also get $\bar{A} \leftrightarrow \bar{B}$ and $\overline{T(A)} \leftrightarrow \overline{T(B)}$, all making one equivalence class of equivalences, so to speak. We will only prove one representative of each class, and mention the results generated by symmetry. Complementatiton works regardless of the shape, so, out of a complementary pp pair $\left(p_{1} \mid p_{2}\right),\left(15-p_{1}, 15-p_{2}\right)$ we will usually prefer the one, whose coordinates sum to less than 15.

\subsection{New patterns and new shapes}

From the computer experiments came several realizations. Firstly, equivalence is not the norm - in the nicest of shapes, the square, there are 15 separate equivalence classes of pps. It is very easy to come up with sensible-looking, topologically connected shapes, in which all pps are inequivalent. Secondly, after accounting for symmetry and complements, there are several equivalences unrelated to the ones above, which hold in nice (e.g. connected, Definition 2.77 shapes. Thirdly, these equivalences, and many more, hold in shapes far less regular than Young diagrams and rectangles. The goal of this paper is to explain, and make precise, as many of these observations as possible.

There is one observation that might be extremely difficult to make rigorous. Given an equivalence, we can prove that it holds in some class of shapes (e.g. connected ones). Empirically, it may seem that it holds in no other shapes (so, the description of the class is tight). However, to say that the equivalence holds if and only if the shape is in the class, we would need to rule out numerical coincidences. The computer experiments did not suggest why this should be true, and indeed, even when our results look tight, it is probably only true in some statistical sense.

\subsection{Plan of attack}

Assuming that "if and only if" characterizations of equivalences are infeasible, the next best thing is to try and prove equivalences with shape hypotheses as weak as we can make them. One can then look at empirical equivalences not explained by the theorems, and use them to produce conjectures with yet weaker hypotheses. So, in a practical sense, the "if and only if" problem may have a solution. We hope that for any interesting class of shapes, one should either be able to find a counterexample, or to prove that the equivalence holds (see, e.g. 3.2.

\subsection{Results}

This paper proves five distinct equivalences, grouped into four sections. An overview of the equivalences, and their symmetries (read Subsection 3.1 first!) is given in Table 1 on page 683. The best way to understand the implications of these results is to read the next subsection, which gives some example applications.

\subsection{Example applications of the results}

Our work strengthens both of the previously known equivalences. Theorem 4.7 proves that the $\left(\begin{array}{l}0 \\ 0\end{array}: 0\right) \leftrightarrow$ $(. .9:$ : :) bijection holds for bottom-right CR-erasable shapes (Definition 4.1); this class includes Young diagrams. Additionally, the first bijection of Section 7 proves that $(.01: 0$ is equivalent to its top-left - 


\begin{tabular}{|c|c|c|c|c|c|c|c|c|}
\hline \multicolumn{3}{|c|}{ Section 5} & \multicolumn{3}{|c|}{ Section 4} & \multicolumn{3}{|c|}{ Section 6} \\
\hline $\begin{array}{l}\left(\begin{array}{l}\bullet \\
0 \\
0 \\
0 \\
0\end{array}\right) \\
(0 \mid 1)\end{array}$ & $\leftrightarrow$ & 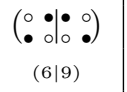 & 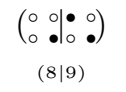 & $\leftrightarrow$ & 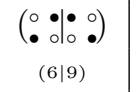 & 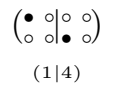 & $\leftrightarrow$ & (::00 \\
\hline 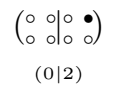 & $\leftrightarrow$ & 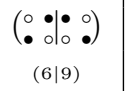 & 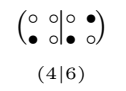 & $\leftrightarrow$ & 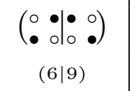 & 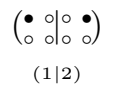 & $\leftrightarrow$ & $\begin{aligned}-{ }^{\circ} \text { old } \\
(6 \mid 9\end{aligned}$ \\
\hline 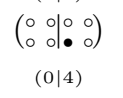 & $\leftrightarrow$ & 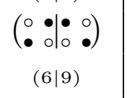 & 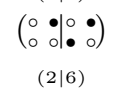 & $\leftrightarrow$ & 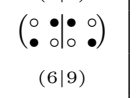 & 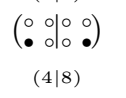 & $\leftrightarrow$ & 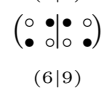 \\
\hline 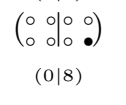 & $\leftrightarrow$ & 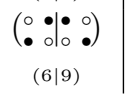 & 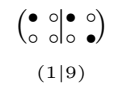 & $\leftrightarrow$ & $\begin{array}{c}\left(\begin{array}{c}0 \\
\bullet\end{array} \mid \begin{array}{l}\bullet \\
0\end{array}\right) \\
(6 \mid 9)\end{array}$ & $\begin{array}{c}\left(\begin{array}{c}0 \\
0 \\
0\end{array} 0 \begin{array}{l}1 \\
0 \\
0\end{array}\right) \\
(2 \mid 8)\end{array}$ & $\leftrightarrow$ & $\left.\begin{array}{c}\left(\begin{array}{c}0 \\
\bullet\end{array}\right. \\
(6 \mid 0 \\
(6 \mid 9)\end{array}\right)$ \\
\hline \multicolumn{9}{|c|}{ Section 7} \\
\hline 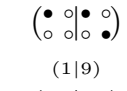 & $\leftrightarrow$ & $\begin{aligned} &\left(\begin{array}{c}0 \\
0\end{array}\right. 0 \\
&(8 \mid 0 \\
&(8 \mid 9)\end{aligned}$ & 121 & $\leftrightarrow$ & 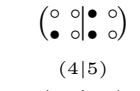 & $\begin{array}{c}\left(\begin{array}{c}\bullet \\
0 \\
0\end{array}\right)\left[\begin{array}{l}0 \\
0\end{array}\right) \\
(1 \mid 5)\end{array}$ & $\leftrightarrow$ & $\begin{array}{r}\left(\begin{array}{l}0 \\
0 \\
0\end{array}\right) \\
(8 \mid 1\end{array}$ \\
\hline 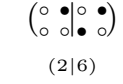 & $\leftrightarrow$ & $\begin{array}{c}\left(\begin{array}{c}0 \\
0\end{array} 0^{\circ}: 0^{0}\right) \\
(4 \mid 6)\end{array}$ & 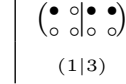 & $\leftrightarrow$ & 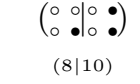 & 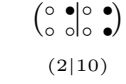 & $\leftrightarrow$ & $\begin{array}{r}\left(\begin{array}{l}0 \\
.0 \\
(4 \mid]\end{array}\right] \\
\text { (4) }\end{array}$ \\
\hline
\end{tabular}

Tab. 1: Pattern pair equivalences described in this paper, by section. In order to make the symmetries clearer, the table does not always show the canonical pp of a complementary pair. For example, we write $\left(\begin{array}{l}0 \\ 0\end{array} 0_{0}^{\bullet} \circ\right)$ instead of the usual $\lrcorner$-diagram $(\stackrel{0}{0}: \bullet: \bullet)$.

bottom-right reflection $(.010$ ) in connected diagrams. Taking the complement, this pp is equivalent to

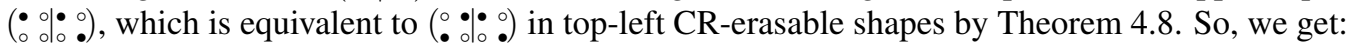

Corollary 3.1. If a shape is bottom-right CR-erasable, or connected and top-left CR-erasable, the pps

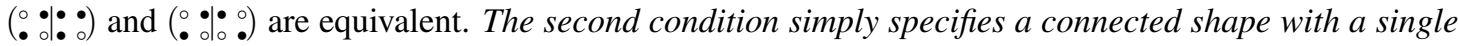
bottom-right corner; this includes all top-left - bottom-right reflections of Young shapes.

This chain of deductions shows that the equivalence holds for many more shapes than previously known. However, the shape hypotheses are also not too nice, and, empirically, not tight. This illustrates two important points: (i) the results in this paper say more about pp equivalences than is apparent at first glance - we intend to present these implications more systematically in a later revision of [7]; (ii) daisy-chaining results as above does not usually give the weakest possible restrictions.

The case of $(.: 0 \mid::) \leftrightarrow(:::::)$, proved by Burstein for Young shapes in [1], is similar. We take the complementary $\left(\begin{array}{c}\circ \\ 0\end{array} 0.0 \%\right.$ right CR-erasable shapes. Again, this does not seem to be tight empirically, but in this case we lost nothing by daisy-chaining, because the theorems have identical hypotheses.

The previous paragraphs prove equivalences in rather broad classes of shapes. The flip side of the coin is to describe equivalences that hold in a specific class of shapes. For example, take Young shapes. They are top-right, bottom-left, and bottom-right CR-erasable. Hence, Theorems 4.7. 5.1 and 6.1 all apply, and

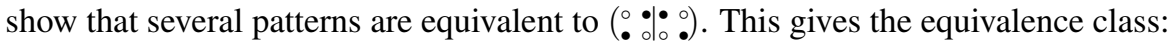

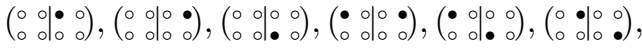

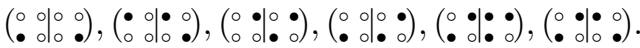


Moreover, the second bijection of Section 7 applies, giving four more classes:

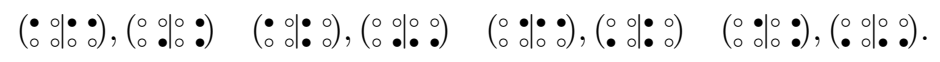

Explicitly computing the pp equivalences for the Young diagram $(6,6,4,2,2,2)$, we found that these are the only equivalence classes.

Corollary 3.2. The above equivalence classes describe all the equivalences that hold for every Young diagram (also, see the note about symmetry in Subsection 3.1).

\section{$4 \quad(: 0 \mid::)$ and $(: 01: \circ):\lrcorner$-diagrams and Acyclic Orientations}

This pair of pps started it all. Their equivalence was first proved by Alex Postnikov [6], using a recurrence for $\lrcorner$-diagrams found by Lauren Williams [10], and his analogous recurrence for acyclic orientations.

\section{$4.1\lrcorner$-diagrams}

Originally, a $\lrcorner$-diagram was defined to be a binary filling of a Young diagram having the $\lrcorner$-property: if two cells contain $\bullet$, and are located at the bottom-left and top-right corners of a rectangle, then the bottomright corner must also contain • (together, these cells look like "」”). In our terminology, a $\lrcorner$-diagram is a $(: 01:: 0$ )-avoiding filling. This definition is valid for all shapes. There is a caveat here: in a Young diagram, one always has the top-left corner of that rectangle. In general, that cell might be missing, but our definition requires it to be present. We chose to have a complete $2 \times 2$ minor because this naturally preserves the connection between acyclic orientations and $\lrcorner$-diagrams - the proof for acyclic orientations requires all four cells to be present.

Lauren Williams introduced the polynomial $F_{S}(q)$, where the coefficient of $q^{k}$ counts the number of $\left(\because:\right.$ : :)-avoiding fillings of shape $S$ that contain $k \bullet$ s. She gave a simple recurrence for $F_{S}(q)$ in Young diagrams. We will show that it generalizes to a much larger class of shapes.

The recurrence starts at a bottom-right corner $c$ of the shape $S$, marked with $*$ in $(1)$ - that is, the cell must be rightmost in its row, and bottommost in its column.

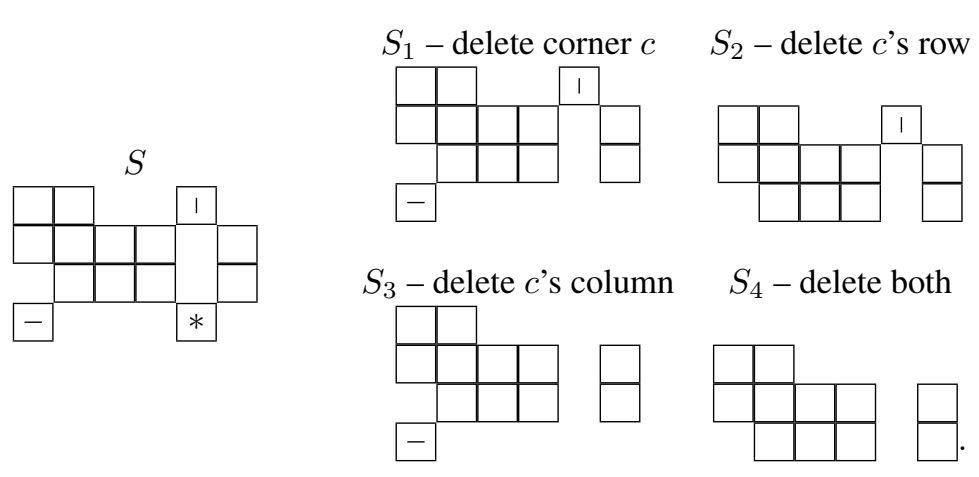

The cells above $c$ and the cells to the left of $c$, ignoring discontinuities, form the bottom and right edges of a rectangle, marked with $\mid$ and - in (1). In order for the recurrence to work, all the cells in this rectangle must be present (this is true for $S$ ). We will call such rectangles complete. Because of Remark 2.11, we will also require the shape to be $2 \times 2$-connected. To compute $F_{S}(q)$, the recurrence requires the values 
of $F_{S}(q)$ on four smaller shapes, as in 11 . These deletions may render the shape $2 \times 2$-disconnected. To compute $F_{S_{i}}$, we will split $S_{i}$ into its $2 \times 2$-connected components $S_{i}^{(j)}$, each a separate shape, and multiply their polynomials:

$$
F_{S_{i}}(q)=\prod_{j} F_{S_{i}^{(j)}}(q)
$$

Some of the components will be detached cells, each of which will contribute a factor of $(1+q)$, because it may be filled with either $\circ$ or $\bullet$, independently of any other cell. In our example, the four shapes simplify to one (modulo detached cells, which are responsible for the $(1+q)^{i}$ factors):

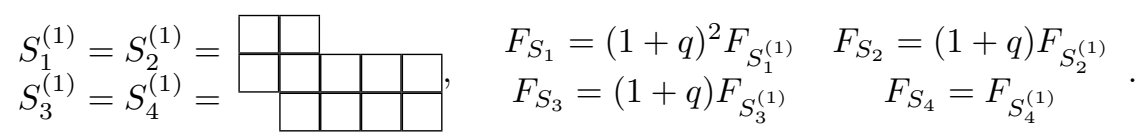

Now, the recurrence (we will define it in Lemma 4.3 may be used to compute $F$ for every $S_{i}^{(j)}$. The decomposition of $S_{i}$ into $S_{i}^{(j)}$ is necessary to cover a larger class of shapes. Without taking $2 \times 2$ components, we cannot apply the recurrence to shapes like (a), or to compute $F$ for shapes like (b) that reduce to (a):

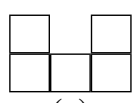

(a)

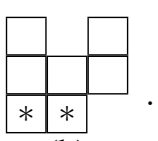

(b)

This points out an important problem: for some shapes, we may be unable to repeatedly apply the recurrence all the way down to the empty shape. The worst case is repeated expansion along $S_{1}$ (delete corner); in order for it to succeed, this definition must apply:

Definition 4.1. A shape is bottom-right complete rectangle-erasable (in the rest of this subsection, we write brCR-erasable) if all of its $2 \times 2$-components satisfy the following recursive rule. In each component $S^{(j)}$, considered as a separate shape, we can find a special bottom-right corner $c$ with two properties. Firstly, the corner must have a complete rectangle. Secondly, the shape $S^{(j)} \backslash c$ must be brCR-erasable. As the base case, the empty shape is brCR-erasable.

Lemma 4.2. If $S^{(j)}$ is a brCR-erasable $2 \times 2$-connected component, use the special corner $c$ to obtain $S_{1}, S_{2}, S_{3}$, and $S_{4}$. Then, all these $S_{i}$ are brCR-erasable $\left(S_{1}\right.$ - by definition, the rest require an easy argument).

We have now completely described what the recurrence needs to compute $F_{S}(q)$. It remains to say how it works:

Lemma 4.3. If the shape $S$ is brCR-erasable, then the generating polynomial of $\left(0_{\bullet}^{\circ}: \bullet\right)$-avoiding fillings of $S$ with $k \bullet$ s can be computed using only the recurrence

$$
F_{S}(q)=\prod_{j}\left(q F_{S_{1}^{(j)}}(q)+F_{S_{2}^{(j)}}(q)+F_{S_{3}^{(j)}}(q)-F_{S_{4}^{(j)}}(q)\right)
$$

where $S^{(j)}$ are the $2 \times 2$-connected components of $S$ considered as separate shapes, and $S_{i}^{(j)}$ are copies of $S^{(j)}$ after deleting the special corner, the cells in its row, column, and row plus column, as discussed above. The initial condition is $F_{\emptyset}(q)=1$. 
Proof. Most of the proof is done already: we saw that $F_{S}$ is a product over $2 \times 2$-connected components, and we showed that the $S_{i}^{(j)}$ are brCR-erasable. It remains to explain the expression inside the parentheses.

Consider a particular $S^{(j)}$; by Definition 4.1 it has a special bottom-right corner $c$ with a complete rectangle (the next cell to be deleted). If the corner contains $\bullet$, then no forbidden pattern can involve this corner (because both patterns have $\circ$ in the bottom-right corner). So, the number of such pafs is $F_{S_{1}^{(j)}}(q)$, and we multiply it by $q$ to account for $\bullet$ in the corner.

If the corner contains $\circ$, this constrains the cells above and to the left of $c$. Since the shape contains $c$ 's complete rectangle, $\bullet$ must not be simultaneously present in both the column above and in the row to the left of $c$. Either $c$ 's row or $c$ 's column must consist entirely of os. If it is the row, then it cannot participate in a forbidden pattern - both patterns have at least one $\bullet$ in each row. The number of ways to fill the remaining cells is enumerated by $F_{S_{2}^{(j)}}(q)$. The reasoning for the column case is identical, and that contributes $F_{S_{3}^{(j)}}(q)$. However, this double-counts the case when both the row and the column are filled with os, so we subtract $F_{S_{4}^{(j)}}(q)$.

\subsection{Acyclic orientations}

Recall from Definition 2.2 that a bipartite graph $G_{S}$ corresponds to each shape $S$. A filling of the shape gives an orientation: the edge points from a row to a column if its cell contains $\circ$, and from a column to a row otherwise. A cycle in this graph corresponds to a sequence of cells in the filling alternating between "same column, different row" and "same row, different column", with contents (independently) alternating between $\circ$ and $\bullet$. Here is an example:
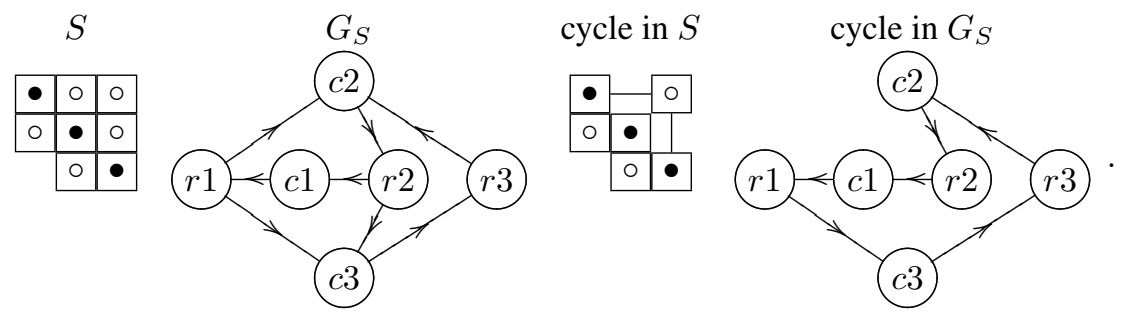

Note that in this example, there is also a 4-cycle: $c 1-r 1-c 2-r 2$, which corresponds to the minor $\{r 1, r 2\} \times\{c 1, c 2\}$ in the filling. In fact, every 4 -cycle is a $2 \times 2$ minor filled with one of the two patterns

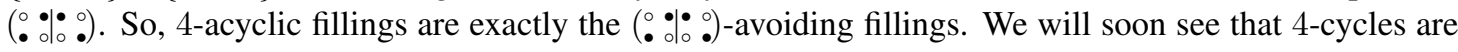
present in every cyclic filling of a large class of shapes. First, recall the notions of "complete rectangle" and bottom-right CR-erasability. Neither depends on having a corner as the special cell, and we will drop this requirement. From now on, a cell $c$ has a complete rectangle if for any two cells $d$ and $e$ in its row and column, the shape contains the fourth cell of the $2 \times 2$ minor containing these three cells. A shape is CR-erasable if it satisfies Definition 4.1, but the special cell need not be a bottom-right corner. By saying top-left CR-erasable we may require it to be that type of corner; we may also require some other type of cell. However, with no qualifiers, any cell is allowed.

Lemma 4.4. Let $S$ be a $2 \times 2$-connected shape with a CR-cell c. Then $S^{\prime}=S \backslash$ c has at most three $2 \times 2$-connected components, all but one of which are detached cells that are leaf edges of $G_{S^{\prime}}$. 
From Lemma 4.4, we see that if a $2 \times 2$-connected shape $S$ is CR-erasable, the deletion procedure is particularly simple. First, we delete some CR-cell from $S$. Then, we delete the resulting detached cells, and we are once again left with a $2 \times 2$-connected CR-erasable shape.

Lemma 4.5. Suppose that $S$ is a $2 \times 2$-connected, CR-erasable shape. Then, any cyclic filling of $S$ contains a 4-cycle.

For shapes satisfying this condition, acyclic orientations are the $\left(0_{0}^{\circ} 0_{0}^{\bullet}\right)$-avoiding fillings. As with $\lrcorner$ diagrams, this pattern-avoidance model is not perfect - in this case, if the shape is arbitrary, we cannot express acyclicity in terms of small minors. For example, one needs a $3 \times 3$ minor to detect a cycle in this shape (see (4)):

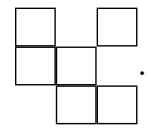

It has $64\left({ }^{\circ} 0^{\bullet}\right.$ •)-avoiding (4-acyclic) fillings, and 62 acyclic ones. It is CR-erasable, but is not $2 \times 2$ connected, and cannot be CR-erased without splitting into $2 \times 2$-components. So, it shows that the extra assumption in Lemma 4.5 is necessary.

\subsection{Recurrence for $(: \circ: \circ:$ )-avoidance}

In Lemma 24.2 of [6], Alex Postnikov proved a recurrence for the chromatic polynomial $\chi_{G_{\lambda}}(t)$ of the graph of a Young diagram. He then specialized it to obtain a recurrence for the number of acyclic orientations of $G_{\lambda}$. We will generalize his result to all CR-erasable shapes. However, the chromatic polynomial does not decompose across $2 \times 2$-components. If it did, the recurrence for the chromatic polynomial would hold for all CR-erasable graphs, and we would get the same recurrence for $\left(\left.\begin{array}{l}\bullet \\ \bullet\end{array}\right|_{\circ} ^{\bullet} \bullet\right)$-avoiding fillings as for acyclic fillings. But, this is impossible as shown by example (5). Therefore, we have to specialize to $(\because \cdot \bullet: \bullet)$-avoidance straight away.

Lemma 4.6. Let $A_{S}$ be the number of $\left(0^{\circ} \cdot 0_{\circ}^{\circ}\right)$-pafs of shape $S$. If the shape $S$ is CR-erasable, then $A_{S}$ can be computed using only the recurrence

$$
A_{S}=\prod_{j}\left(A_{S_{1}^{(j)}}+A_{S_{2}^{(j)}}+A_{S_{3}^{(j)}}-A_{S_{4}^{(j)}}\right),
$$

where $S^{(j)}$ are the $2 \times 2$-connected components of $S$ considered as separate shapes, and $S_{i}^{(j)}$ are copies of $S^{(j)}$ after deleting the special cell, the cells in its row, column, and row plus column, just like in Lemma 4.3. The initial condition is $A_{\emptyset}=1$.

Proof. We need to show that for every $2 \times 2$-connected shape $S^{(j)}$ with a CR-cell $c$, the number of $(0)$-pafs is given by the quantity in the parentheses of $(6)$. The rest comes together just as in Lemma 4.3.

By Lemma 4.5 it is enough to compute the number of acyclic fillings of $S^{(j)}$. By Postnikov's Lemma 24.2 [6], the chromatic polynomial $\chi_{S^{(j)}}$ of $G_{S^{(j)}}$ can be written as

$$
\chi_{S^{(j)}}(t)=\chi_{S_{1}^{(j)}}(t)-\frac{1}{t}\left(\chi_{S_{2}^{(j)}}(t)+\chi_{S_{3}^{(j)}}(t)-\chi_{S_{4}^{(j)}}(t)\right) .
$$


Technically, Postnikov's proof was written for a corner of a Young shape, not a CR-cell of a $2 \times 2$ connected shape. However, he uses only the structure of $G_{S}$, and discards the positions of rows and columns. Therefore, it generalizes without modifications to any shape with a CR-cell. Further following Postnikov, we specialize (7) to $t=-1$, to obtain a relation in terms of the numbers of acyclic orientations $(-1)^{n} \chi_{S^{(j)}}(-1)$. The exponent $n$ is the number of vertices in the graph of $S^{(j)}$, and because we only delete the edges, the graph of $S_{i}^{(j)}$. So, if $a o_{S}$ is the number of acyclic fillings of shape $S$, we get

$$
a o_{S^{(j)}}=a o_{S_{1}^{(j)}}+a o_{S_{2}^{(j)}}+a o_{S_{3}^{(j)}}-a o_{S_{4}^{(j)}}
$$

That is not quite the end — we need to show that acyclic fillings and $(0 \%$ sub-shapes $S_{i}^{(j)}$ (for the left-hand side, we know this already). For $S_{1}^{(j)}$, look back at the Lemma 4.4 The shape has one large $\mathrm{CR}$-erasable $2 \times 2$-connected component, and at most two leaf edges. No cycle can pass through leaf edges, and the big component is okay by Lemma 4.5 .

The proofs for $S_{2}^{(j)}, S_{3}^{(j)}$, and $S_{4}^{(j)}$ are slight modifications of the same argument, detailed in [7]. Briefly, the shape remains $2 \times 2$-connected after these whole-row or whole-column deletions, and the proof that the new shape is CR-erasable is just like Lemma 4.2. Thus, Lemma 4.5 applies.

\subsection{Equivalence of $(: \circ \mid: \circ)$ and $(: \circ \mid \circ)$, and its symmetries}

Following [6], we now specialize [3] with $q=1$ to get a recurrence counting the number of $\lrcorner$-diagrams of shape $S$ :

$$
\lrcorner_{S}=\prod_{j}(\lrcorner_{S_{1}^{(j)}}+\right\lrcorner_{S_{2}^{(j)}}+\right\lrcorner_{S_{3}^{(j)}}-\right\lrcorner_{S_{4}^{(j)}}\right) .
$$

This holds for all bottom-right CR-erasable shapes. Such shapes are, of course, CR-erasable, and so the $\left(\begin{array}{l}0.0 \\ 0\end{array}.\right)$ recurrence $(6)$ also applies. The recurrences are identical, and have the same initial conditions, \lrcorner$_{\emptyset}=A_{\emptyset}=1$. To summarize:

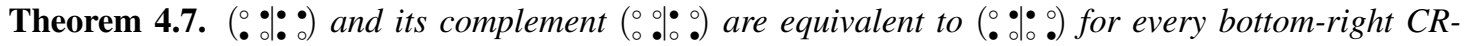
erasable shape.

The recurrence for $(: 0 \mid: 0)$ depends on the bottom-right corner because the pattern pair has an asymme-

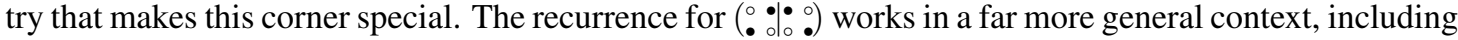
any corner CR-erasability. If we rotate $(.: 0 .: 0)$, a different corner type will become special. Then, we can rotate the proofs and definitions of Subsection 4.1 to obtain some symmetric results. The reader may wish to learn to read upside-down and sideways before continuing.

Theorem 4.8. Omitting complements, the analogs of Lemma 4.3 (8), and Theorem 4.7 hold for bottom-

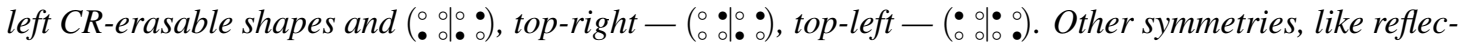
tions, add no further pps.

Combining these theorems with a Section 7 result, we can further weaken the conditions for the

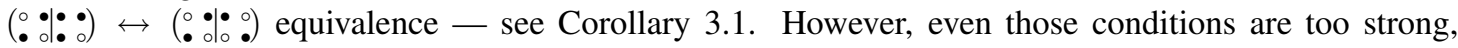
and exclude many empirically equivalent shapes. 


\section{5 ( $(\because \circ): \circ)$ and $(: \circ \mid: \circ)$ : The Same Recurrence}

$\left(\begin{array}{ll}0 \\ 0\end{array}\right)$

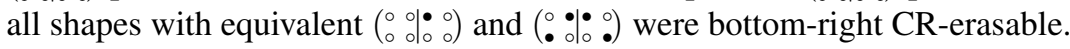

Theorem 5.1. Omitting complements, the following pps are equivalent to $\left(\begin{array}{l}0 \\ 0\end{array} l_{\circ}^{\circ}\right):$ for bottom-right corner CR-erasable shapes - $\left(\begin{array}{lll}0 & 0 \\ 0 & 0\end{array}\right)$

\section{$6 \quad(\because \circ: \circ)$ and $(: \circ 1 \% \circ)$ : The Same Recurrence With a Twist}

The same recurrence can be extended to $\left({ }_{0}^{\circ} o_{\bullet}^{\circ} \circ\right)$, but since this pp is invariant under row swaps, the assumptions are weaker - we can delete CR-cells that are rightmost in their rows. In our experiments,

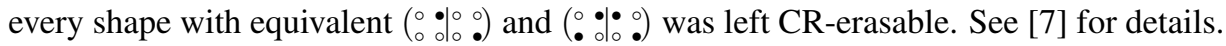

Theorem 6.1. Omitting complements, these pps are equivalent to $\left({ }^{\circ}:\left.\right|_{\circ} ^{\circ}\right)$ : for right CR-erasable shapes

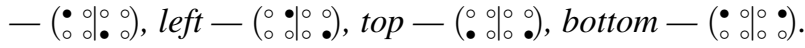

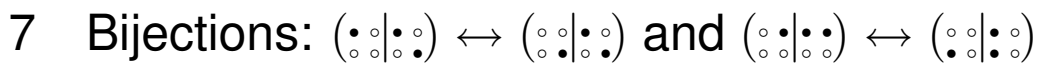

If a shape's $2 \times 2$-connected components are horizontally- and vertically-connected (Definition 2.7), there are two nice, but very different bijections that prove the stated equivalences ${ }^{(i)}$ In both cases, the obvious

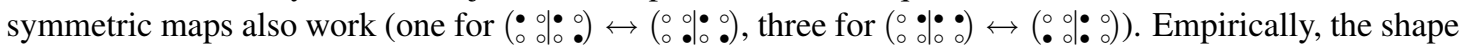
assumptions look tight for both bijections.

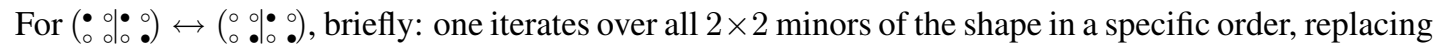
the first instance of the new forbidden pattern (e.g. $\left.\left(\begin{array}{l}\bullet \\ 0 \\ 0\end{array}\right)\right)$ ) by the old forbidden pattern (e.g. $\left(\begin{array}{l}0 \\ 0 \\ 0\end{array}\right)$ ), then restarting the iteration, and repeating until no replacement can be made. Minors $\left(i_{1}, j_{1}\right) \times\left(i_{2}, j_{2}\right)$ should be ordered as follows: first, the minor "size" $\left(i_{2}-i_{1}, j_{2}-j_{1}\right)$ increases in this order:

$$
(1,1),(1,2),(2,1),(1,3),(2,2),(3,1),(1,4), \ldots
$$

Second, for equal sizes, the top-left corner must increase in that same order.

For $\left(\begin{array}{c}0 \\ 0\end{array} 0\right.$

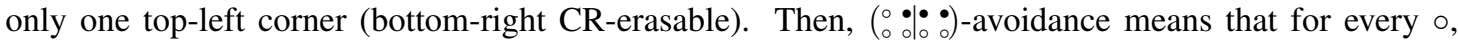
either the row above it contains only os, or the column to the left of it contains only $\bullet$ s (exchange rows

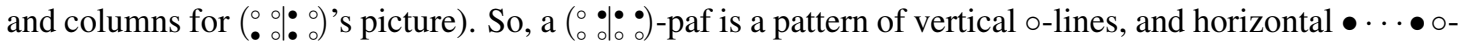
lines. Every row and column contains a line (possibly of zero length). The line lengths describe the filling completely: any cell not in a line is filled by $\bullet$. There are a few easy restrictions on how the lines may intersect; in particular, lines do not cross, and horizontal lines may touch vertical lines, but not vice versa.

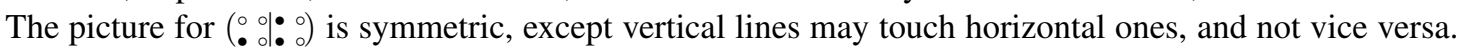

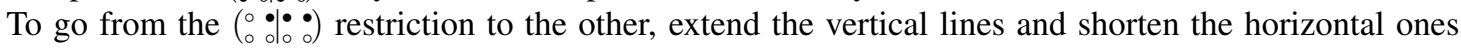
by one cell each (with the obvious inverse). For edge cases, wrap around: $0 \leftrightarrow$ the length of the row or column. Dropping the single-corner condition makes the bijection far more complex.

For the details of both bijections, see [7].

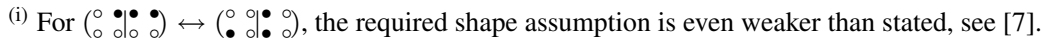




\section{Acknowledgments}

I would like to thank my advisor, Alex Postnikov, for suggesting this problem to me, for his insightful questions and ideas for new directions, and last, but not least, for making me write it all down. I would like to thank Matthieu Josuat-Vergès for his stimulating letters; they helped make this paper better in more ways than I can list here. I would also like to thank Leonid Chindelevitch for translating the abstract into French — he did a far better job than I could. Finally, I would like to thank the reviewers for suggesting several valuable improvements.

\section{References}

[1] A. Burstein, On some properties of permutation tableaux, Annals of Combinatorics 11 (2007), 355-368. arXiv:math/0703004v1.

[2] V. Jelínek: Pattern-avoiding fillings of rectangular shapes, Formal Power Series and Algebraic Combinatorics Nankai University, Tianjin, China, 2007, available at http: / / www. fpsac . cn/ PDF-Proceedings/Posters/47.pdf.

[3] M. Josuat-Vergès, Bijections between pattern-avoiding binary fillings of Young diagrams, February 1, 2008 (in preparation).

[4] S. Kitaev: On Multi-avoidance of Right Angled Numbered Polyomino Patterns, INTEGERS: Electronic Journal of Combinatorial Number Theory 4 (2004), A21.

[5] S. Kitaev, T. Mansour, A. Vella: Pattern Avoidance in Matrices, Journal of Integer Sequences 8 (2005), Article 05.2.2.

[6] A. Postnikov: Total positivity, Grassmannians, and networks, arXiv:math/0609764, but we cite the up-to-date version from http://www-math.mit.edu/ apost/papers.html, downloaded on November 14, 2007, when the arXiv copy was only version 1.

[7] A. Spiridonov: Pattern-Avoidance in Binary Fillings of Grid Shapes, available at http:// www-math.mit.edu/ lesha/papers/pattern-pair-avoidance.pdf (will be updated with new results in April-May, 2008; see revision history).

[8] Z. Stankova: Shape-Wilf-Ordering on Permutations of Length 3, Electronic J. Combin. 14 (2007), no. $1, \mathrm{R} 56$.

[9] E. Steingrímsson and L. Williams, Permutation tableaux and permutation patterns, J. Combinatorial Theory A 114 (2007), No. 2, 211-234. arXiv:math/0507149v2.

[10] L. Williams: Enumeration of totally positive Grassmann cells, Advances in Mathematics 190 (2005), no. 2, 319-342. math.CO/0307271. 